\title{
Subcellular localization of chlorophyllase2 reveals it is not involved in chlorophyll degradation during senescence in Arabidopsis thaliana
}

\author{
Hu, Xueyun ; Jia, Ting ; Hörtensteiner, Stefan ; Tanaka, Ayumi ; Tanaka, Ryouichi
}

\begin{abstract}
Chlorophyllase (CLH), which catalyzes the release of the phytol chain from chlorophyll (Chl), has been long considered to catalyze the first step of Chl degradation. Arabidopsis contains two isoforms of CLH (CLH1 and CLH2), and CLH1 was previously demonstrated to be localized in tonoplast and endoplasmic reticulum, and not be involved in Chl degradation. In contrast, CLH2 possesses a predicted signal-peptide for chloroplast localization, and phylogenetic analysis of CLHs in Arabidopsis and other species also indicate that CLH2 forms a different clade than CLH1. Therefore, the possibility remains that CLH2 is involved in the breakdown of Chl. In the current study, clh mutants lacking CLH2 or both CLH isoforms were analyzed after the induction of senescence. Results indicated that the clh knockout lines were still able to degrade $\mathrm{Chl}$ at the same rate as wild-type plants. Transgenic Arabidopsis plants were generated that constitutively expressed either CLH2 or CLH2 fused to a yellow fluorescent protein (YFP). Observations made using confocal microscopy indicated that CLH2-YFP was located external to chloroplasts. Additionally, in overexpression plants, CLH2 was enriched in tonoplast and endoplasmic reticulum fractions following membrane fractionation. Based on the collective data, we conclude that CLH2 is not involved in Chl breakdown during senescence in Arabidopsis.
\end{abstract}

DOI: https://doi.org/10.1016/j.plantsci.2019.110314

Posted at the Zurich Open Repository and Archive, University of Zurich

ZORA URL: https://doi.org/10.5167/uzh-186606

Journal Article

Accepted Version

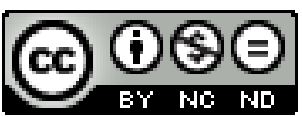

The following work is licensed under a Creative Commons: Attribution-NonCommercial-NoDerivatives 4.0 International (CC BY-NC-ND 4.0) License.

Originally published at:

Hu, Xueyun; Jia, Ting; Hörtensteiner, Stefan; Tanaka, Ayumi; Tanaka, Ryouichi (2020). Subcellular localization of chlorophyllase 2 reveals it is not involved in chlorophyll degradation during senescence in Arabidopsis thaliana. Plant Science, 290:110314.

DOI: https://doi.org/10.1016/j.plantsci.2019.110314 


\title{
Subcellular localization of chlorophyllase2 reveals it is not involved in chlorophyll degradation during senescence in Arabidopsis
}

\section{thaliana}

\author{
Xueyun $\mathrm{Hu}^{1}$, Ting $\mathrm{Jia}^{3}$, Stefan Hörtensteiner ${ }^{4}$, Ayumi Tanaka ${ }^{2}$, Ryouichi Tanaka ${ }^{2 *}$ \\ ${ }^{1}$ College of Bioscience and Biotechnology, Yangzhou University, Yangzhou, 225009, China \\ ${ }^{2}$ Institute of Low Temperature Science, Hokkaido University, N19W8, Kita-ku, Sapporo 060-0819, Japan \\ ${ }^{3}$ Joint International Research Laboratory of Agriculture and Agri-Product Safety of the Ministry of Education \\ of China, Yangzhou University, Yangzhou, 225009, China \\ ${ }^{4}$ Department of Plant and Microbial Biology, University of Zurich, Zollikerstrasse 107 CH-8008 Zurich, \\ Switzerland
}

\begin{abstract}
Chlorophyllase (CLH), which catalyzes the release of the phytol chain from chlorophyll (Chl), has been long considered to catalyze the first step of Chl degradation. Arabidopsis contains two isoforms of CLH (CLH1 and CLH2), and CLH1 was previously demonstrated to be localized in tonoplast and endoplasmic reticulum, and not be involved in Chl degradation. In contrast, CLH2 possesses a predicted signal-peptide for chloroplast localization, and phylogenetic analysis of CLHs in Arabidopsis and other species also indicate that CLH2 forms a different clade than CLH1. Therefore, the possibility remains that CLH2 is involved in the breakdown of Chl. In the current study, clh mutants lacking CLH2 or both CLH isoforms were analyzed after the induction of senescence. Results indicated that the $c l h$ knockout lines were still able to degrade $\mathrm{Chl}$ at the same rate as wild-type plants. Transgenic Arabidopsis plants were generated that constitutively expressed either CLH2 or CLH2 fused to a yellow fluorescent protein (YFP). Observations made using confocal microscopy indicated that CLH2-YFP was located external to chloroplasts. Additionally, in overexpression plants, CLH2 was enriched in tonoplast and endoplasmic reticulum fractions following membrane fractionation. Based on the collective data, we conclude that CLH2 is not involved in Chl breakdown during senescence in Arabidopsis.
\end{abstract}

Keywords: Chlorophyllase2; Chlorophyll breakdown; Senescence; Localization 


\section{Introduction}

Chlorophyll (Chl), the most abundant photosynthetic pigment, is the key component responsible for harvesting solar energy in photosynthetic antenna systems in green plants, and for charge separation and electron transport within photosynthetic reaction centers. Chl and its derivatives, however, are also strong photosensitizers and potentially toxic to cells if they generate excessive levels of reactive oxygen species (ROS) [1]. This suggests that it would be beneficial for chlorophyll to be promptly catabolized to non-photosensitizing forms when leaves begin to senesce [2]. Whether only one or multiple Chl degradation pathways exist in plants is currently under debate. Recent progress on Chl degradation has identified the major Chl degradation pathway. In this pathway, magnesium $(\mathrm{Mg})$ is de-chelated from $\mathrm{Chl} a$ by $\mathrm{Mg}$-dechelatase, encoded by Mendel's green cotyledon gene, STAY-GREEN (SGR), which first converts Chl $a$ to pheophytin $a$ (Phein $a)$ [3]. The phytol chain is then removed by pheophytinase (PPH) from Phein $a$ to produce pheophorbide $a$ (Pheide $a$ ) [4]. Subsequently, the porphyrin ring of Pheide $a$ is oxygenolytically opened by Pheide $a$ oxygenase (PAO), resulting in a loss of the green color of the molecule. The intermediate product, red $\mathrm{Chl}$ catabolite $(\mathrm{RCC})$, is then degraded by a reductase (RCCR). The resulting product, a primary fluorescent $\mathrm{Chl}$ catabolite ( $\mathrm{pFCC}$ ), is modified and ultimately converted to nonfluorescent phyllobilins that are stored in the vacuole [2], [5].

In addition to the major $\mathrm{Chl}$ degradation pathway, there is a possibility that an alternative Chl degradation pathway may exist. This presumption is based on the observation that some level of Chl degradation occurs in both SGR and PPH knockout mutants [6], [7], [4]. The first step in this postulated pathway is removal of the phytol chain, i.e. conversion of $\mathrm{Chl}$ to phytol and chlorophyllide (Chlide) a by chlorophyllase (CLH) activity, followed by magnesium (Mg) dechelation from Chlide $a$ by Mg-dechelatase; the latter of which is suggested to be catalyzed by SGR-like (SGRL) [3].

CLH activity was discovered more than 100 years ago [8]. There are two isoforms of CLH (AtCLH1 and AtCLH2) in Arabidopsis. It has been suggested that neither of the CLH isoforms are involved in Chl degradation during senescence based on the observation that absence of these CLHs does not affect dark-induced senescence, and that overexpressed CLH-GFP fusion proteins are detected outside chloroplasts in plant cells [9]. Further analysis of CLH1 localization indicated that it is localized in the endoplasmic reticulum (ER) and the tonoplast [10]. Thus, it was concluded 
that CLH1 is not involved in chlorophyll degradation. Recently, a PPH homologue, CHLOROPHYLL DEPHYTYLASE1 (CLD1), was hypothesized to be responsible for dephytylation of Chl $a$ for the recycling of chlorophyll molecules during the repair of photosystem II [11]. Additionally, CLD1 was also suggested to be involved in Chl degradation; however, overexpression of CLD1 did not result in a detectable level of Chl degradation in transgenic plants [11]. This raises the question: Are AtCLHs actually involved in Chl catabolism? Although AtCLH1 was demonstrated not to be involved in chlorophyll degradation [10], the possibility still remains that AtCLH2 may be involved in Chl turnover or degradation.

Several lines of evidence indicated that AtCLH2 may function in chlorophyll degradation. Phenotypic analysis of clh2 mutants indicated that they exhibit a slight delay in Chl and LHCII degradation; indicating that CLH2 functions in catalyzing Chl degradation [9]. Additionally, although AtCLH2-GFP was previously observed to be localized outside of chloroplasts, those observations may not accurately reflect the localization of native AtCLH2 as the GFP tag may alter the protein [12]. A proteomic analysis also detected AtCLH1, but not AtCLH2, in vegetative vacuoles, and microsomal, tonoplast and lipid droplet samples [13], [14], [15]; suggesting that AtCLH2 may be located inside chloroplasts. AtCLH2 also possesses a typical but putative chloroplast transit peptide sequence [9]. Lastly, the discovery of a chloroplast enzyme named SGR-like that converts Chlide $a$ to Pheide $a$ opens up the possibility that CLH2 may contribute to Chl degradation [3]. Among all of this supportive evidence, intracellular localization of CLH2 is key to understanding the function of AtCLH2 as part of an alternative pathway for chlorophyll degradation. Thus far, it appears that the localization of AtCLH2 has only been investigated using CLH2-GFP [9] and it is known that the addition of an artificial tag may alter the targeting and localization of the fusion protein. Therefore, it is necessary to check the localization of native AtCLH2, without the addition of any artificial tag sequences, to demonstrate the actual localization of AtCLH2.

In the present study, our objective was to examine the subcellular localization of the native form of AtCLH2 and determine if AtCLH2 functions in Chl degradation or turnover in vivo. Observations of the phenotype of single and double knockout mutants of AtCLHs indicated that no significant effect on Chl breakdown during dark-induced senescence could be detected in the CLH2 mutant lines relative to their respective wild-type plants. Furthermore, we demonstrated through the use of isolated organelles and membrane fractions, that native AtCLH2 is located in 
both the tonoplast and the ER. Collectively, the data indicate that AtCLH2 is not involved in Chl metabolism in Arabidopsis. The probable physiological role of AtCLH2 is discussed.

2. Materials and methods

\subsection{Plant material}

The Arabidopsis T-DNA insertion line FLAG_076H05 [designated clh2-1; ecotype Wassilewskija $(W s)$ ] and $\operatorname{clh} 1-1 / 2-1$, described by Schenk et al. [9] was used in the current study along with wildtype $W s$ and Columbia $(\mathrm{Col})$. Plants were grown in soil under long days (16 h light/8 h dark) or short days (10 h light/14 $\mathrm{h}$ dark) in growth chambers under fluorescent lights (70$90 \mu \mathrm{mol}$ photons $\mathrm{m}^{-2} \mathrm{~s}^{-1}$ ) at $23{ }^{\circ} \mathrm{C}$ for 4 weeks. To conduct the senescence experiments, leaves with three biological replicate from each long day-grown plant were detached and placed on wet filter paper (3 mM MES buffer, pH 5.8, with or without $50 \mu \mathrm{M}$ MeJA) and incubated in complete darkness for up to 4 days.

\subsection{Pigment analysis}

Leaves were imaged with a digital camera and leaf area was calculated using image $\mathbf{J}$ software (https://imagej.nih.gov/ij/). Pigments were extracted from leaf tissues by homogenization in precooled acetone at $-30{ }^{\circ} \mathrm{C}$ as described by $\mathrm{Hu}$ et al. [16] . Extracts were centrifuged for 5 min at $15,000 \times \mathrm{g}$ at $4{ }^{\circ} \mathrm{C}$, and the supernatant was analyzed by HPLC using a Symmetry C8 column (150 $\mathrm{mm}$ in length, $4.6 \mathrm{~mm}$ in i.d.; Waters, Milford, MA, USA) according to the method of Zapata et al. [17]. Elution profiles were monitored by measuring absorbance at $410 \mathrm{~nm}$. Standard pigments (Chl $a$ and Chl $b$ ) were purchased from Juntec Co. Ltd. (Odawara, Japan).

\subsection{Arabidopsis transformation}

AtCLH2 was cloned from complementary DNA (cDNA) of wild-type Col. YFP was cloned from the pEarleyGate101 plasmid by PCR [18] . The C-terminal fusion (AtCLH2-YFP) construct was generated by fusing the YFP sequence to the C-terminus of AtCLH2, and then the 5'UTR and 3'UTR of AtCLH2 were fused to the $\mathrm{N}$ and C-terminus of the fusion sequence, respectively. The fusion sequence and cDNA of $A t C L H 2$ without any tag were put into pENTR4-Dual. The construct was cloned into pENTR using a LR clonase reaction and transferred into the expression vector, pEarleyGate100, containing a gene conferring glufosinate (Basta) resistance [18]. The constructs were confirmed by sequencing. The recombinant binary vectors containing AtCLH2-YFP and 
AtCLH2 were introduced into Agrobacterium (Rhizobium radiobacter) strain GV2260 and GV3101, respectively. Arabidopsis was transformed using the floral dip method [19] and twoweek-old seedlings of putative transformants were selected with Basta to identify positive transformants.

\subsection{Localization of AtCLH2-YFP with confocal microscopy}

Protoplasts were isolated, as described by Robert et al. [20], from fully-expanded leaves of fourweek-old plants grown under long-day conditions. Expression and localization of AtCLH2-YFP protein in transgenic plants was determined by confocal fluorescence microscopy (Nikon DEclipse C1si confocal microscope, Nikon, Japan) using a $488 \mathrm{~nm}$ excitation wavelength and emission wavelengths of 496 to $706 \mathrm{~nm}$ were collected.

\subsection{Separation of membrane and soluble fractions}

Leaves from four-week-old plants grown under short days were harvested and lysed in liquid nitrogen using a mortar and pestle. The fractions were prepared as previously described [21]. Briefly, leaves were homogenized in a mixture containing $100 \mathrm{mM}$ MOPS-NaOH (pH 7.0), $1 \mathrm{mM}$ $\mathrm{MnCl}_{2}$, and a protease inhibitor cocktail (Sigma), and then centrifuged at 3,000 $\times \mathrm{g}$ for 10 min at $4^{\circ} \mathrm{C}$. Supernatants were subsequently centrifuged at $160,000 \times \mathrm{g}$ for $60 \mathrm{~min}$ at $4^{\circ} \mathrm{C}$ to generate a membrane (pellet) fraction and a soluble (supernatant) fraction.

\subsection{Isolation of intact chloroplasts and vacuoles}

Rosette leaves were obtained from four-week-old plants grown under short days. Intact chloroplasts were prepared according to the method described by Salvi et al.,[22]. Briefly, leaves were homogenized in isolation buffer containing $20 \mathrm{mM}$ Tricine-NaOH (pH 8.0), 0.45 M sorbitol, $10 \mathrm{mM}$ EDTA, $1 \mathrm{mM} \mathrm{NaHCO}$, 0.1\% (w/v) BSA, $0.05 \%$ (w/v) DTT, and $0.05 \%$ (w/v) PVP. The homogenate was filtered through four layers of Miracloth and then centrifuged at 1,000 $\times \mathrm{g}$ for 5 min. The pellet was suspended in a wash buffer containing $20 \mathrm{mM}$ MOPS-NaOH (pH 7.6), 0.33 M sorbitol, $5 \mathrm{mM} \mathrm{MgCl}$, and $2.5 \mathrm{mM}$ EDTA. After a Percoll density gradient centrifugation, intact chloroplasts were collected from the interface between $40 \%$ and $80 \%$ Percoll. The intact chloroplast suspension was washed twice and then the chloroplasts were osmotically ruptured in a swelling buffer containing $10 \mathrm{mM}$ MOPS-NaOH (pH 7.6) and $4 \mathrm{mM} \mathrm{MgCl}$. Intact vacuoles were prepared as described by Robert et al. [20]. Fully-expanded leaves were cut into $2 \mathrm{~mm}$ strips and 
placed in a protoplast enzyme solution containing 0.4 M mannitol, $10 \mathrm{mM}$ MES (pH 5.6), $27 \mathrm{mM}$ $\mathrm{CaCl}_{2}, 1 \%(\mathrm{w} / \mathrm{v})$ cellulose, $1 \%(\mathrm{w} / \mathrm{v})$ macerozyme, and $0.035 \%(\mathrm{v} / \mathrm{v}) \beta$-mercaptoethanol. A vacuum was applied for $10 \mathrm{~min}$, and then the leaf strips were incubated in the dark at room temperature on a rotary shaker at $70 \mathrm{rpm}$ for another $4 \mathrm{~h}$. The released protoplasts were passed through a $150 \mu \mathrm{m}$ mesh filter and centrifuged at $80 \times \mathrm{g}$ for $20 \mathrm{~min}$ at $20^{\circ} \mathrm{C}$. Protoplasts were washed two times in a washing buffer (0.4 M mannitol, $10 \mathrm{mM} \mathrm{MES}, \mathrm{pH}$ 5.6) and then resuspended in $10.5 \mathrm{ml}$ pre-warmed $\left(37^{\circ} \mathrm{C}\right)$ lysis buffer $(0.2 \mathrm{M}$ mannitol, $10 \%$ (w/v) Ficoll, 10 mM EDTA, $5 \mathrm{mM}$ sodium phosphate, $\mathrm{pH} 8.0$ ). After $5 \mathrm{~min}, 3 \mathrm{ml}$ of the solution was overlaid with $3 \mathrm{ml} 4 \%$ (w/v) Ficoll solution and $1 \mathrm{ml}$ ice-cold vacuole buffer (0.2 M mannitol, 2 mM EDTA, 5 $\mathrm{mM}$ sodium phosphate, $\mathrm{pH} 7.5$ ). The gradient was centrifuged at $71,000 \times \mathrm{g}$ for $50 \mathrm{~min}$ at $10^{\circ} \mathrm{C}$. Vacuoles were visible as a pink layer at the interface between 10 and $4 \%$ Ficoll. Protein concentrations were determined by the Lowry method [23], using bovine serum albumin (BSA) as a protein standard.

\subsection{Membrane fractionation}

Arabidopsis leaves used for membrane fractionation were grown in soil for $28 \mathrm{~d}$ under short-day conditions. The utilized fractionation method was previously described with some modifications [21], [24]. Leaf tissues were homogenized in a buffer containing $50 \mathrm{mM}$ Tris (pH 7.6), 20\% (v/v) glycerol, and $150 \mathrm{mM} \mathrm{NaCl}$ with either $5 \mathrm{mM} \mathrm{MgCl}_{2}$ and $2 \mathrm{mM}$ EGTA (for subsequent sucrose (Suc) gradient separation in the presence of $\mathrm{Mg}^{2+}$ ) or with $2 \mathrm{mM}$ EDTA (for subsequent Suc gradient separation in the absence of $\mathrm{Mg}^{2+}$ ). Leaves were homogenized in a blender (Waring Laboratory \& Science) three times at low speed using a 10-fold greater amount of buffer than sample. Homogenates were filtered through two layers of Miracloth (Merck) and then centrifuged for $10 \mathrm{~min}$ at $10,000 \times \mathrm{g}$ at $4^{\circ} \mathrm{C}$. Supernatants were ultra-centrifuged for $30 \mathrm{~min}$ at $100,000 \times \mathrm{g}$ at $4{ }^{\circ} \mathrm{C}$. The resulting pellets were resuspended $(0.5 \mathrm{~mL} / \mathrm{g})$ in a buffer containing $50 \mathrm{mM}$ MOPS$\mathrm{NaOH}(\mathrm{pH} 7.0), 10 \%(\mathrm{w} / \mathrm{v})$ Suc, EDTA-free complete protease inhibitor cocktail (Roche Diagnostics $\mathrm{GmbH}$ ), and either no $\mathrm{MgCl}_{2}$ or $5 \mathrm{mM} \mathrm{MgCl}_{2}$. The suspension was then centrifuged at $6000 \times \mathrm{g}$ for $3 \mathrm{~min}$ to remove unsuspended small pellets. A total of $600 \mu \mathrm{l}$ of the supernatant was loaded on top of the following discontinuous Suc gradient (from bottom to top): $200 \mu 1$ of $55 \%$ Suc, $450 \mu \mathrm{l}$ of $50 \%, 45 \%, 40 \%, 35 \%, 30 \%, 25 \%$, and $20 \%$ Suc solution along with the same components as the resuspension buffer. The gradient was centrifuged in a bucket rotor (Beckman 
TLA100.2) for $20 \mathrm{~h}$ at $100,000 \times \mathrm{g}$ at $4{ }^{\circ} \mathrm{C}$. After centrifugation, the gradient was collected in 200 $\mu \mathrm{l}$ increment fractions from top to bottom, and the fractions were stored at $-30{ }^{\circ} \mathrm{C}$ until used in the immunoblot analysis.

\subsection{Immunoblot analysis}

Total protein was extracted from leaves using 10 volumes $(\mathrm{v} / \mathrm{w})$ of protein extraction buffer containing $50 \mathrm{mM}$ Tris- $\mathrm{HCl}(\mathrm{pH}$ 8), 12\% (w/v) Suc, 2\% (w/v) lithium lauryl sulfate, and $1.5 \%$ $(w / v)$ dithiothreitol. Prior to SDS-PAGE separation, protein extracts were mixed with an equal vol. of $2 \times$ Ling's urea buffer containing $10 \mathrm{mM}$ Tris- $\mathrm{HCl}$ (pH 8.0), 10\% (w/v) Suc, 2\% (w/v) SDS, 1 mM EDTA, 4 mM dithiothreitol, a small amount of bromophenol blue, and $10 \mathrm{M}$ urea. Proteins were separated on a $14 \%(\mathrm{w} / \mathrm{v})$ polyacrylamide gel and electroblotted to polyvinylidene difluoride membranes. For immunoblots with the subfractions of cells, the following amounts of proteins were loaded: membrane and soluble fractions and vacuole samples, $4 \mu \mathrm{g}$ of proteins; leaf and chloroplast samples, $16 \mu \mathrm{g}$ of protein. For immunoblots with membrane fractions, the same volume of each fraction was loaded on a 14\% polyacrylamide gel. AtCLH2 protein was detected with anti-AtCLH2 antiserum produced in rabbits against recombinant Arabidopsis CLH2 expressed in Escherichia coli. The type of membrane in the immunoblot was determined using a set of commercial polyclonal rabbit antibodies (Agrisera) against specific markers: ER luminal BiP2 (Agrisera), Plasma membrane (PM) marker $\mathrm{H}^{+}$-ATPase (Agrisera), tonoplast marker VPPase (Cosmo Bio, Inc.). Thylakoid membranes were identified by HPLC analysis of Chl content [17].

\subsection{Phylogenetic analysis}

The protein sequences used for phylogenetic analysis were aligned using CLUSTAL W [25]. The alignment was further optimized by manual inspection and curation. The phylogenetic tree was generated with the MEGA X program, using the neighbor-joining method [26]. All gaps and missing data in the alignments were accounted for by pairwise deletion. Branch points were tested for significance by bootstrapping with 1000 replications.

\section{Results:}

\section{Phylogenetic analysis of CLHs}


A total of 25 previously-identified CLHs from 14 plant species were subjected to phylogenetic analysis to examine the evolutionary relationships between different CLHs (Figure S1). The analysis indicated that CLH1 and CLH2 sequences formed distinct clades, indicating that CLH2s are phylogenetically distinct from CLH1s. Thus, we hypothesized that CLH2 may have a different sub-cellular localization and function than CLH1s.

\section{clh2 knockout lines degrade $\mathrm{Chl}$ at the same rate as wild-type (Ws) plants during dark- induced senescence}

In a previous study, a slight inhibition in Chl degradation was observed in $\operatorname{clhl} / \mathrm{clh} 2$ double knockout lines and in a clh2-1single knockout line, but not in $\operatorname{clh} 1-1$ and $\operatorname{clh} 2-2$ single mutants [9]. The difference between the rate of chlorophyll degradation during dark-induced senescence in the clh1-1/2-2 double knockout mutant and the Col wild type ecotype, however, was not significantly different; regardless if the dark-treated leaves were also exposed to methyl-jasmonate (MeJA) [10]. Since the clh2-1 mutant was derived from a Ws background, and the phenotype and leaf characteristics of $\operatorname{clh} 1-1 / \operatorname{clh} 2-1$ are more similar to Ws than to Col, we compared the rate of Chl degradation of the $c l h 2-1$ mutant with the Ws wild ecotype (Fig 1A).

All of the leaves from four-week-old plants grown under long-day conditions were detached and placed in darkness for $4 \mathrm{~d}$. Leaves of $\operatorname{clh} 2-1$ and $\operatorname{clh} 1-1 / 2-1$, as well as the wild-type, turned yellow during the dark incubation. The average $\mathrm{Chl}$ content per unit area of leaves collected before and after the dark incubation was measured to quantify and compare changes in Chl content in the different lines (Fig. 1). Results indicated that Chl degradation was not inhibited in clh2-1 mutants. The decrease in Chl content in $\operatorname{clh} 2-1$ and $\operatorname{clh} 1-1 / \operatorname{clh} 2-1$ leaves in response to the dark incubation was similar to the level of degradation observed in Ws leaves. The decrease in Chl content in clh21 and $\operatorname{clh} 1-1 / \operatorname{clh} 2-1$ leaves was also similar to Ws leaves when both were exposed to MeJA during the dark period. Previously, however, the decrease in Chl content in $\operatorname{clh} 2-1, \operatorname{clh} 1-1 / 2-1$ was reported to be less than the level of degradation observed in $\mathrm{Col}$ during dark incubation [9]. Since the genetic background of clh2-1 is Ws, results of our study clearly indicate that the rate of Chl degradation in $\operatorname{clh} 2-1$ and $\operatorname{clh} 1-1 / 2-1$ leaves was similar to $W s$ leaves.

\section{AtCLH2-YFP is located outside the chloroplast}


AtCLH2-YFP (C-terminal fusion) was over-expressed in Arabidopsis to determine its subcellular localization. Protoplasts were isolated from leaves of lines in which AtCLH2-YFP was stably overexpressed and examined with confocal microscopy. It was clear, based on the observation of the fluorescent signals, that the fluorescence of YFP (green) was located outside the chloroplast (red), and thus supposedly located in the cytosol (Fig. 2). This observation is in agreement with the results of a transient expression experiment reported by Schenk et al. [9]. As previously indicated, however, it is possible that the fusion of YFP or GFP to CLH2 may affect its intracellular localization.

\section{AtCLH2 is localized to both tonoplast and ER membranes}

The subcellular localization of native AtCLH2 (without any modification) was examined in Arabidopsis to determine if the localization of the AtCLH2 using confocal microscopy was affected by either the YFP (current study) or GFP tag [12]. Total protein extracts from Col leaves were prepared and the level of AtCLH2 was examined by immunoblotting using anti-CLH2 antibody. Results indicated, however, that the level of AtCLH2 was below the detection limit. Therefore, in order to detect native AtCLH2 and to avoid potential interference of AtCLH1 in the immuno-detection, AtCLH2 was overexpressed in a clh1-1 background in which AtCLH1 is lacking. Membrane and soluble fractions were prepared from CLH2-OX overexpressing lines and subjected to immunoblot analysis. Results indicated that AtCLH2 was mainly detected in the membrane fraction with very little detected in the soluble fraction (Fig. 3). The marker proteins, BiP2 for ER and V-PPase for the tonoplast, were also mainly detected in the membrane fraction; although a minor amount of $\mathrm{BiP} 2$ was also detected in the soluble fraction. These results indicate that AtCLH2 may be localized to ER membranes.

Subsequently, both intact chloroplasts and vacuoles were isolated from leaves of the $c l h 1-1$ mutant and $\mathrm{CLH} 2-\mathrm{OX}$ overexpressing line, and were used to determine localization of AtCLH2 by immunoblotting. AtCLH2 was concentrated in the vacuole fraction of CLH2-OX, while AtCLH2 was barely detected in the chloroplast fraction (Fig. 3). This result indicates that AtCLH2 is localized to the tonoplast and not the chloroplast (Fig. 3). A low level of signal for both BiP2 and CLH2 were detected in the chloroplast fractions; however, we speculate that this was due to a low level of contamination of the chloroplast preparations with ER membranes resulting in faint immunoblot signals from ER-located CLH2 in this fraction. 
To further confirm the tonoplast (and possible ER) localization of AtCLH2, membranes were fractionated by sucrose (Suc) density gradient centrifugation. The distribution of CLH2 and marker proteins in the fractionated membranes was then analyzed by immunoblotting. Most of AtCLH2 immunoblot signal was detected in fractions No.11-15 when $\mathrm{Mg}^{+}$was not present in the buffer used in the Suc density gradient. This recognition profile was most similar to profile obtained for the tonoplast marker V-PPase (most appeared in fraction No. 9-15) (Fig. 4).

Subsequently, total subcellular fractions were obtained from leaves using buffer containing $\mathrm{Mg}^{2+}$. Association of ribosomes with the ER is $\mathrm{Mg}^{2+}$-dependent, i.e. presence of $\mathrm{Mg}^{2+}$ in the extraction, and centrifugation gradient buffers results in an association of ribosomes with the ER and a diagnostic redistribution of ER from lower to higher density in the Suc gradient [27], [28]. Results indicated that the position of the membrane-bound fraction of the ER marker BiP2 had shifted to heavier positions when the addition of $\mathrm{Mg}^{2+}$ was used in the extraction buffer. Whereas, the pattern obtained for the markers of the plasma membrane and the tonoplast were largely independent of the presence of $\mathrm{Mg}^{2+}$ ions, with only a slight shift observed from lower to higher density fractions of the gradient (Fig. 4). Relative Chl content, used as a marker for chloroplast thylakoid membranes, also did not exhibit a significant shift. Notably, the position of AtCLH2 in the gradient also exhibited an obvious shift. When $\mathrm{Mg}^{2+}$ was used in the extraction buffer and thus present in the Suc density gradient, the peak of the AtCLH2 immunoblot signal shifted to fraction No.15; which was much different from the fraction exhibiting the highest V-PPase immunoblot signal (fraction No. 11), but overlapping with fraction containing the peak immunoblot signal of BiP2. These results suggest that, in addition to the tonoplast, some AtCLH2 is also localized to the ER. Collectively, the data indicate that AtCLH2 is mainly localized to tonoplast and partly to the ER.

\section{Discussion:}

We previously demonstrated that AtCLH1 is localized to the tonoplast and ER, and that it is not involved in chlorophyll degradation during leaf senescence [10]. In the present study, a phylogenetic analysis revealed that CLH2 sequences from different plant species are phylogenetically distinct from CLH1s (Figure S1). We also determined that, although CLH2 has a predicted transit peptide sequence for the chloroplast, it is localized to the tonoplast and ER and is not involved in Chl degradation during senescence. 
The phenotype of $\operatorname{clh} 2-1$ and the $\operatorname{clh} 1-1 / 2-1$ double mutant did not exhibit any inhibition of chlorophyll degradation during dark-induced senescence, compared to the phenotype of wild type Ws plants, either in the absence or presence of MeJA (Fig. 1). Based on the obtained data, we conclude that AtCLH2 is not involved in Chl degradation in either dark-induced or MeJA-induced senescence.

CLH2-RNAi plants have been reported to exhibit a decreased level of Chlide without a substantial change in the total amount of extractable Chl. As a result, the CLH2-RNAi plants have a lower ration of Chlide to Chl in their leaves [29]; suggesting that AtCLH2 may be involved in Chl turnover, but not in Chl degradation. For example, AtCLH was hypothesized to function in a salvage pathway for Chlide [30]. That study indicated, however, that neither AtCLH1 or AtCLH2 are involved in this pathway [30]. To further explore if AtCLH2 is involved in Chl metabolism in the current study, the subcellular localization of AtCLH2 was investigated, since it has been hypothesized that $\mathrm{Chl}$, the proposed substrate for $\mathrm{CLH} 2$, is degraded to a colorless intermediate inside the chloroplast [31]. Previous research demonstrated that the properties of proteins fused to fluorescent protein tags may be altered from the native form of the protein, leading to aberrant localization, aggregation, and metabolic alterations [12]. Interestingly, when senescent mesophyll protoplasts were used for examining the localization of the green fluorescence of AtCLH2-GFP that was transiently expressed, it was sometimes found in cytoplasmic vesicles [9]. Similarly, we also observed the presence of AtCLH2-YFP in cytoplasmic vesicles in the current study (Fig. S2), possibly reflecting the aberrant localization and/or aggregation of AtCLH2-YFP. Therefore, it was important to determine the subcellular location of native AtCLH2 without any modifications.

Our results indicate that the protein level of AtCLH2 in wild-type leaves was below a level that could be detected with anti-CLH2 antibody. Therefore, native AtCLH2 was overexpressed in wildtype plants to determine the subcellular localization of AtCLH2. Results indicated that, like AtCLH1 [10], AtCLH2 was also localized outside of chloroplasts. More specifically, AtCLH2 appears to be localized to tonoplast and ER membranes (Fig 3 and 4). CLH was found as a glycoprotein in the diatom, Phaeodacylum tricornutum [32], [33] and native CLH in Chenopodium album ( $\mathrm{CaCLH}$ ) was assumed to be modified by glycosylation at an Asn residue. This observation suggests that a modification (glycosylation) occurs in the ER, since the ER is a major site for the glycosylation of proteins. Tsuchiya and coworkers unsuccessfully used heterologous screening of a cDNA library in an attempt to isolate $\mathrm{CaCLH}$, the gene encoding chloroplast-type CLH in 
Chenopodium album [34]. CaCLH possesses a signal sequence at the $\mathrm{N}$ terminus for the ER, however, no sequence is present for ER retention. Since 11 amino acid residues exist between the putative ER cleavage site and the $\mathrm{N}$ terminus of the putative mature $\mathrm{CaCLH}$, it was assumed that $\mathrm{CaCLH}$ is transported to other organelles, such as the vacuole [34]. Because of the similar subcellular localization of AtCLH1 and AtCLH2, it is possible that both of them are transported to the tonoplast via the ER, although no predicted signal sequence for ER localization is present at the $\mathrm{N}$ terminus. Many proteins, however, locate to the ER despite the lack of a signal peptide. For example, phenylalanine ammonia lyase (PAL1), which is involved in lignin biosynthesis, was demonstrated to be associated with ER membranes despite not having a predicted ER signal sequence [35].

It was also suggested that Chl may be transported outside of chloroplasts and is then degraded by (extra-plastidial) AtCLH [36]. Chl, however, appears to be degraded to a colorless intermediate inside of chloroplasts [2], except for a few situations in tobacco and Arabidopsis where some Chl or its green derivatives are detected in vacuoles [37], [38], [39]. Chl $a$ in tobacco was detected in 'senescence-associated vacuoles' (SAVs) in cells of leaves senescing at a relatively slow rate in non- ethephon treated leaves but not in cells of ethephon-treated leaves; suggesting the existence of an alternative non-plastid pathway for Chl degradation that occurs within SAVs only under certain conditions [37]. More recently, in vitro incubation of isolated SAVs in darkness has been recently reported to initially cause a substantial increase in the level of Chlide $a$ [39], implying that CLHs may be involved in the process of Chl degradation that takes place in SAVs. Small chloroplasts exhibiting Chl autofluorescence were detected in Arabidopsis inside vacuoles when senescence was rapidly induced in individually darkened leaves [38]. Similarly, entire photodamaged chloroplasts with $\mathrm{Chl}$ autofluorescence were observed to be transported to the central vacuole by autophagy, and absent in autophagy-impaired mutants [40]. How Chl is degraded inside of a vacuole is still unknown. Considering the relatively high activity of CLHs, and that the level of $\mathrm{Chl}$ and Chlide $a$, the major pigments in SAVs, increase slowly during the incubation of isolated SAVs [39], it seems plausible that CLHs are not involved in autophagymediated Chl degradation. Instead, it is more likely that CLHs are involved in other processes, such as the rapid dephytylation of $\mathrm{Chl}$ in response to herbivore attack [10]. 


\section{Acknowledgements:}

The authors would like to thank Dr. Tohru Tsuchiya for providing us with the anti-CLH2 antibody. This work was supported by the Japan Society for the Promotion of Science [KAKENHI grant No. 16H06554 to R.T.], China Postdoctoral Science Foundation [Grant No. 2018M630608 to X.H.], the Swiss National Science Foundation [Grant No. 31003A_172977 to S.H.], the natural Science Foundation of Jiangsu Higher Education Institutions of China [Grant No. 18KJB210011 to T.J.], the Innovative and Entrepreneurial Talent of Jiangsu Province, and "Lvyang Jinfeng" talents attracting plan.

\section{Author contributions}

X.H. and R.T. planned the experiments. X.H. and T.J. executed the experiments while A.T. and R.T. provided guidance. X.H., T.J., S.H., A.T., R.T. together prepared the manuscript. All authors read and approved the manuscript.

\section{Declaration of interests}

The authors declare no competing interests.

\section{References:}

[1] R.G.L. op den Camp, D. Przybyla, C. Ochsenbein, C. Laloi, C. Kim, A. Danon, D. Wagner, É. Hideg, C. Göbel, I. Feussner, M. Nater, K. Apel, Rapid induction of distinct stress responses after the release of singlet oxygen in Arabidopsis, Plant Cell. 15 (2003) 23202332.

[2] S. Hörtensteiner, Chlorophyll degradation during senescence., Annu. Rev. Plant Biol. 57 (2006) 55-77.

[3] Y. Shimoda, H. Ito, A. Tanaka, Arabidopsis STAY-GREEN, Mendel's green cotyledon gene, encodes magnesium-dechelatase, Plant Cell. 28 (2016) 2147-2160.

[4] S. Schelbert, S. Aubry, B. Burla, B. Agne, F. Kessler, K. Krupinska, S. Hörtensteiner, Pheophytin pheophorbide hydrolase (pheophytinase) is involved in chlorophyll breakdown during leaf senescence in Arabidopsis., Plant Cell. 21 (2009) 767-785.

[5] M. Hauenstein, B. Christ, A. Das, S. Aubry, S. Hörtensteiner, A role for TIC55 as a hydroxylase of phyllobilins, the products of chlorophyll breakdown during plant senescence, Plant Cell. 28 (2016) 2510-2527. 
[6] G. Ren, K. An, Y. Liao, X. Zhou, Y. Cao, H. Zhao, X. Ge, B. Kuai, Identification of a novel chloroplast protein AtNYE1 regulating chlorophyll degradation during leaf senescence in Arabidopsis, Plant Physiol. 144 (2007) 1429-1441.

[7] Y. Sato, R. Morita, M. Nishimura, H. Yamaguchi, M. Kusaba, Mendel's green cotyledon gene encodes a positive regulator of the chlorophyll-degrading pathway, Proc. Natl. Acad. Sci. USA. 104 (2007) 14169-14174.

[8] A. Stoll, Über chlorophyllase und die chlorophyllide, Ph.D. thesis, Nr. 49, ETH Zürich, 1912., (n.d.).

[9] N. Schenk, S. Schelbert, M. Kanwischer, E.E. Goldschmidt, P. Dörmann, S. Hörtensteiner, The chlorophyllases AtCLH1 and AtCLH2 are not essential for senescence-related chlorophyll breakdown in Arabidopsis thaliana., FEBS Lett. 581 (2007) 5517-5525.

[10] X. Hu, S. Makita, S. Schelbert, S. Sano, M. Ochiai, T. Tsuchiya, S.F. Hasegawa, S. Hörtensteiner, A. Tanaka, R. Tanaka, Reexamination of chlorophyllase function implies its involvement in defense against chewing herbivores, Plant Physiol. 167 (2015) 660-670.

[11] Y.-P. Lin, M.-C. Wu, Y. Charng, Identification of a chlorophyll dephytylase involved in chlorophyll turnover in Arabidopsis, Plant Cell. 28 (2016) 2974-2990.

[12] S.K. Tanz, I. Castleden, I.D. Small, A.H. Millar, Fluorescent protein tagging as a tool to define the subcellular distribution of proteins in plants., Front. Plant Sci. 4 (2013) 214.

[13] C. Carter, S. Pan, J. Zouhar, E.L. Avila, T. Girke, N. V Raikhel, The vegetative vacuole proteome of Arabidopsis thaliana reveals predicted and unexpected proteins., Plant Cell. 16 (2004) 3285-3303.

[14] S.M. Zargar, R. Kurata, S. Inaba, A. Oikawa, R. Fukui, Y. Ogata, G.K. Agrawal, R. Rakwal, Y. Fukao, Quantitative proteomics of Arabidopsis shoot microsomal proteins reveals a cross-talk between excess zinc and iron deficiency, Proteomics. 15 (2015) 1196-1201.

[15] L. Brocard, F. Immel, D. Coulon, N. Esnay, K. Tuphile, S. Pascal, S. Claverol, L. Fouillen, J.-J. Bessoule, C. Bréhélin, Proteomic analysis of lipid droplets from Arabidopsis aging leaves brings new insight into their biogenesis and functions, Front. Plant Sci. 8 (2017) 894.

[16] X. Hu, A. Tanaka, R. Tanaka, Simple extraction methods that prevent the artifactual conversion of chlorophyll to chlorophyllide during pigment isolation from leaf samples., Plant Methods. 9 (2013) 19.

[17] M. Zapata, F. Rodríguez, J.L. Garrido, Separation of chlorophylls and carotenoids from marine phytoplankton, a new HPLC method using a reversed phase C8 column and phridine-containing mobile phases, Mar. Ecol. Prog. Ser. 195 (2000) 29-45.

[18] K.W. Earley, J.R. Haag, O. Pontes, K. Opper, T. Juehne, K. Song, C.S. Pikaard, Gatewaycompatible vectors for plant functional genomics and proteomics, Plant J. 45 (2006) 616629.

[19] Clough SJ, Bent AF, Floral dip: a simplified method for Agrobacterium-mediated transformation of Arabidopsis thaliana, Plant J. 16 (1998) 735-743.

[20] S. Robert, J. Zouhar, C. Carter, N. Raikhel, Isolation of intact vacuoles from Arabidopsis rosette leaf-derived protoplasts., Nat. Protoc. 2 (2007) 259-262. 
[21] T. Oka, F. Saito, Y. Shimma, T. Yoko-o, Y. Nomura, K. Matsuoka, Y. Jigami, Characterization of endoplasmic reticulum-localized UDP-D-galactose: hydroxyproline Ogalactosyltransferase using synthetic peptide substrates in Arabidopsis., Plant Physiol. 152 (2010) 332-340.

[22] D. Salvi, N. Rolland, J. Joyard, M. Ferro, Purification and proteomic analysis of chloroplasts and their sub-organellar compartments, in: Organ Proteomics.Methods Mol. Biol., 2008: pp. 19-36.

[23] O.H. Lowry, N.J. Rosebrough, A.L. Farr, R.J. Randall, Protein measurement with the Folin phenol reagent, J. Biol. Chem. 193 (1951) 265-275.

[24] K. Wulfetange, S.N. Lomin, G.A. Romanov, A. Stolz, A. Heyl, T. Schmülling, The cytokinin receptors of Arabidopsis are located mainly to the endoplasmic reticulum, (2011) Plant Physiol 156: 1808-1818.

[25] J.D. Thompson, D.G. Higgins, T.J. Gibson, CLUSTAL W: improving the sensitivity of progressive multiple sequence alignment through sequence weighting, position-specific gap penalties and weight matrix choice, Nucleic Acids Res. 22 (1994) 4673-4680.

[26] S. Kumar, G. Stecher, M. Li, C. Knyaz, K. Tamura, MEGA X: Molecular evolutionary genetics analysis across computing platforms, Mol. Biol. Evol. 35 (2018) 1547-1549.

[27] J. Lord, T. Kagawa, T. Moore, H. Beevers, Endoplasmic reticulum as the site of lecithin formation in castor bean endosperm, J. Cell Biol. 57 (1973) 659-667.

[28] J.M. Lord, Isolation of endoplasmic reticulum: General principles, enzymatic markers, and endoplasmic reticulum-bound polysomes, Methods Enzymol. 148 (1987) 576-584.

[29] Y. Liao, K. An, X. Zhou, W.J. Chen, B.K. Kuai, AtCLH2, a typical but possibly distinctive chlorophyllase gene in Arabidopsis, J. Integr. Plant Biol. 49 (2007) 531-539.

[30] Y.P. Lin, T.Y. Lee, A. Tanaka, Y.Y. Charng, Analysis of an Arabidopsis heat-sensitive mutant reveals that chlorophyll synthase is involved in reutilization of chlorophyllide during chlorophyll turnover, Plant J. 80 (2014) 14-26.

[31] S. Hörtensteiner, B. Kräutler, Chlorophyll breakdown in higher plants, Biochim. Biophys. Acta - Bioenerg. 1807 (2011) 977-988.

[32] W. Terpstra, Identification of chlorophyllase as a glycoprotein, FEBS Lett. 126 (1981) 231235.

[33] W. Terpstra, J. Lambers, Y. Levine, Studies on chlorophyllase: I. Release of the enzyme from photosynthetic membranes of Phaeodacylum tricornutum, Photobiochem Photobiophys. 11 (1986) 249-255.

[34] T. Tsuchiya, H. Ohta, K. Okawa, A. Iwamatsu, H. Shimada, T. Masuda, K. Takamiya, Cloning of chlorophyllase, the key enzyme in chlorophyll degradation: Finding of a lipase motif and the induction by methyl jasmonate, Proc. Natl. Acad. Sci. U. S. A. 96 (1999) 15362-15367.

[35] J.E. Bassard, J. Mutterer, F. Duval, D. Werck-Reichhart, A novel method for monitoring the localization of cytochromes $\mathrm{P} 450$ and other endoplasmic reticulum membrane associated proteins: A tool for investigating the formation of metabolons, FEBS J. 279 (2012) 1576-1583. 
[36] K.I. Takamiya, T. Tsuchiya, H. Ohta, Degradation pathway(s) of chlorophyll: what has gene cloning revealed?, Trends Plant Sci. 5 (2000) 426-431.

[37] D.E. Martínez, M.L. Costa, F.M. Gomez, M.S. Otegui, J.J. Guiamet, "Senescenceassociated vacuoles" are involved in the degradation of chloroplast proteins in tobacco leaves., Plant J. 56 (2008) 196-206.

[38] S. Wada, H. Ishida, M. Izumi, K. Yoshimoto, Y. Ohsumi, T. Mae, A. Makino, Autophagy plays a role in chloroplast degradation during senescence in individually darkened leaves, Plant Physiol. 149 (2009) 885-893.

[39] F.M. Gomez, C.A. Carrión, M.L. Costa, C. Desel, T. Kieselbach, C. Funk, K. Krupinska, J.J. Guiamét, Extra-plastidial degradation of chlorophyll and photosystem I in tobacco leaves involving "senescence-associated vacuoles," Plant J. (2019) on line. doi:10.1111/tpj.14337.

[40] M. Izumi, H. Ishida, S. Nakamura, J. Hidema, Entire photodamaged chloroplasts are transported to the central vacuole by autophagy, Plant Cell. 29 (2017) 377-394.

\section{Legends to the Figures}

Figure 1. Chlorophyll degradation in leaves of $\operatorname{clh} 2-1$, $\operatorname{clh} 1-1 / \operatorname{clh} 2-1$ and wild type (Ws) plants during dark-induced senescence. Chlorophyll levels in leaves of four-week-old plants after $4 \mathrm{~d}$ of dark-induced leaf senescence in the absence (B and E) or presence $(\mathrm{C}$ and $\mathrm{F})$ of $50 \mu \mathrm{M} \mathrm{MeJA}$, compared to chlorophyll levels prior to the induction of leaf senescence (A and D). Leaf images are shown at top (A-C), and chlorophyll levels of the corresponding leaves are shown at the bottom (D-F). Leaf number refers to oldest (bottom of plant) to youngest (top of plant) leaves. The oldest (Nos. 1 and 2) and youngest (Nos. 9 and 10) leaves were pooled prior to chlorophyll extraction and chlorophyll measurement. Data represent the mean $\pm \operatorname{SD}(n=3)$.

Figure 2. Subcellular localization of CLH2-yellow fluorescent protein (YFP) fusion protein (green fluorescence). Protoplasts were prepared from leaves of plants constitutively expressing CLH2-YFP. Red fluorescence is chlorophyll autofluorescence.

Figure 3. Subcellular localization of $\mathrm{CLH} 2$ in isolated organelles obtained from leaves of Arabidopsis after $28 \mathrm{~d}$ of growth. Leaf tissue from $\mathrm{CHL2}-\mathrm{OX} / \mathrm{clh} 1-1$ plants was fractionated into membrane (P10 and P100), soluble (S100), chloroplast and vacuole fractions for the analysis of CLH2 localization. Total leaf, chloroplast, and vacuole fractions were prepared from leaves of both chl1-1 and CHL2-OX/clh1-1 (CLH2-OX) plants. A total of 4, 16, 16 and $4 \mu \mathrm{g}$ of protein were used from membrane/soluble, leaf, chloroplast, and vacuolar samples, respectively, and 
loaded on an SDS-PAGE gel. The blotted membrane was then immunologically probed with antiCLH2, anti-V-PPase, anti-LHCI type I chlorophyll $a / b$-binding protein (Lhca1), anti-luminalbinding protein2 (BiP2) and anti-Rubisco large subunit antisera.

Figure 4. Localization of $\mathrm{CLH} 2$ in fractionated membranes from leaves of $C H L 2-O X / \mathrm{clh} 1-1$ plants grown for 28 days. Cellular membranes were separated into 20 fractions by sucrose density gradient centrifugation of microsomal fractions in the presence $\left(+\mathrm{Mg}^{2+}\right)$ or absence of $\mathrm{Mg}^{2+}(-$ $\mathrm{Mg}^{2+}$ ). Odd-numbered fractions were analyzed by immunoblotting with anti-CLH2, anti- $\mathrm{H}^{+}-$ ATPase, anti-BiP2, and anti-V-PPase antibodies. Thylakoid membrane concentrations are represented by relative chlorophyll $a$ (Chl a) content in each fraction. PM, Plasma membrane. ER, endoplasmic reticulum. 
A
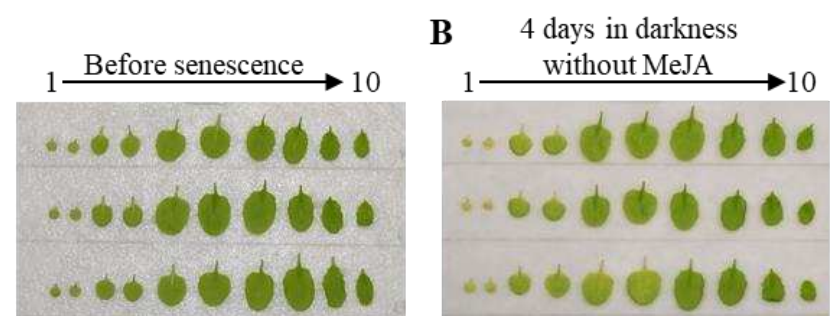

C 4 days in darkness

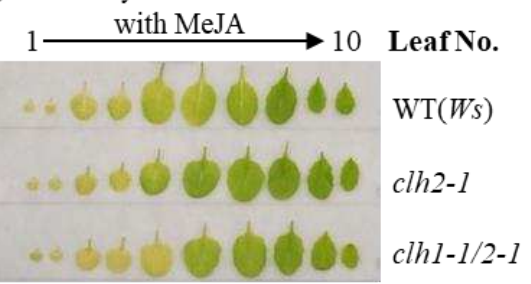

D

E
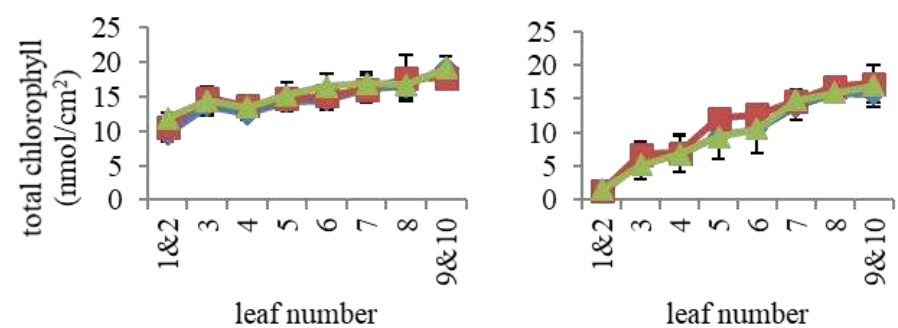

leaf number

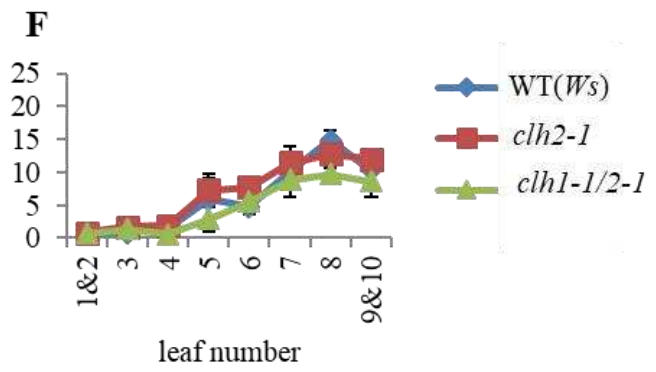

Figure 1 (color should be used) 


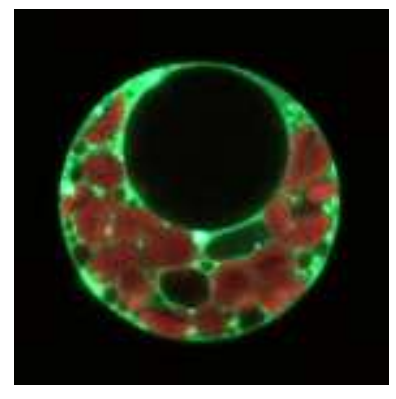

Figure 2 (color should be used) 


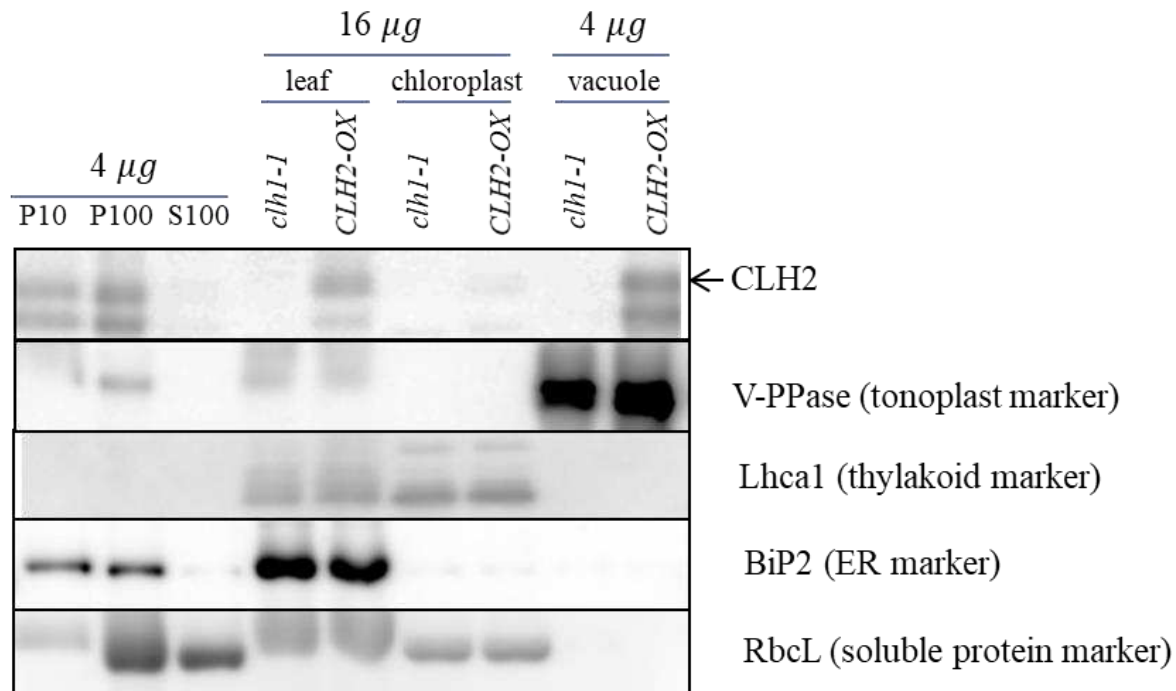

Figure 3 


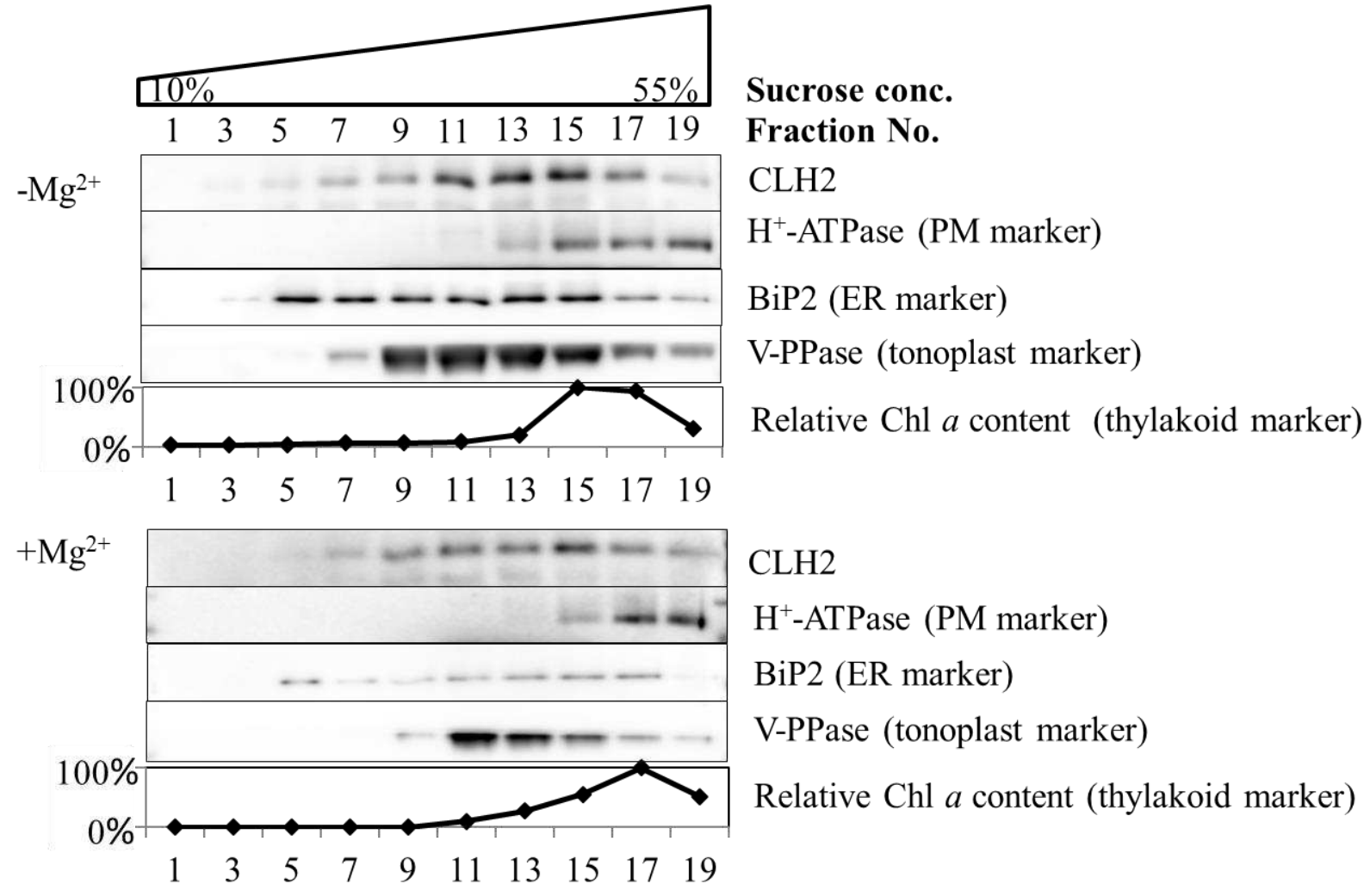

Figure 4 